Forum Kind Jugend Sport $2021 \cdot 2: 150-151$ https://doi.org/10.1007/s43594-021-00053-6 Angenommen: 8. Oktober 2021

Online publiziert: 5. November 2021

(c) Der/die Autor(en) 2021

\section{Originalpublikation}

Morsbach, K., Edelhoff, D., Brockers, P., Gebken, U. (2021). Open Sunday. Konzepte und Einblicke in eine sport- und sozialpädagogische Arena für alle Kinder. Hildesheim: arete Verlag.

Wer jemals den Versuch unternommen hat, ein erfolgreiches, langfristiges und über die Sozialraumgrenzen hinaus bekanntes sportpädagogisches Projekt Sozialer Arbeit umzusetzen, findet sich in diesem Buch (• Abb. 1) vollständig wieder. Die Autoren ${ }^{\star}$ innen des „Open Sunday" berichten sehr detailreich, wie es ein anfänglich kleines Projekt mit der ersten Umsetzung 2013 geschafft hat, mittlerweile über 20.000 Kinder und Jugendliche in rund einem Dutzend deutscher Kommunen erreicht zu haben.

Nicht benutzte Sporthallen an einem „offenen Sonntag“ zu nutzen, ist keine Idee der Autoren ${ }^{*}$ innen, sondern - als eine Art sozialwissenschaftliche FranchiseUnternehmung - die stetig weiterentwickelte Idee einer Züricher Stiftung. Deren Grundgedanke war und ist es, Bewegung, Spiel und Sport für alle Kinder anzubieten. Da der Zusammenhang von exklusiver Sportvereinszugehörigkeit und Kindern aus sozial höher gestellten Familien bekannt ist, liegt die Zielsetzung des Open Sunday in niederschwelligen Angeboten und Gelegenheiten für sechsbis zwölfjährige Mädchen und Jungen aus sozial herausfordernden Stadtteilen. Was der Begriff "chancenbenachteiligt“ in der Realität bedeutet, schildert nicht nur Kap. 2, welches theoretisch fundiert die großen gesellschaftlichen Herausforderungen sozialer Ungleichheit aufzeigt.

\title{
Rolf Schwarz
}

Institut für Bewegungserziehung und Sport, Pädagogische Hochschule Karlsruhe, Karlsruhe, Deutschland

\section{Der offene Sonntag prosperiert}

\section{Eine Betrachtung des sportpädagogischen Projekts Open Sunday: viel Lob und eine wunde Stelle}

Beeindruckend, geradezu beklemmend erweist sich Abschn. 3.4 mit der Beschreibung der kindlichen Bewegungswelt in Duisburg-Hochfeld. Spätestens hier zeigen die Autoren*innen, warum der Ansatz sportpädagogisch geprägter Sozialer Arbeit für die Übernahme sozialer Verantwortung so wichtig ist und eine eigenständige Forschungspraxis legitimiert.

Da die Bildungs- und Entwicklungschancen vieler Kinder und Jugendlichen in Deutschland teilweise eklatant minimiert sind, nimmt sich das Buch nachvollziehbar viel Zeit und Raum zu erklären, wie es das Projekt überhaupt schafft, bei diesem abgehängten Klientel Anschluss zu finden. Der in der Literatur Sozialer Arbeit mittlerweile häufig zu findende Fachterminus der Niederschwelligkeit, der ähnlich inflationär benutzt wird wie der Begriff der Nachhaltigkeit, erhält im Open Sunday eine ausführliche, verständliche und sehr konkrete Operationalisierung: kostenloser Einlass, unmittelbare Sozialraumnähe (keine Mobilitätsbarrieren), bekannte und vertraute Räumlichkeiten, keine elterliche Begleitung nötig, keine normierten Sportarten, stattdessen freie Spielformen („polysportiv“) und Bewegungslandschaften, keine Bürokratie, jedes Geschlecht ist willkommen, keine sprachlichen oder religiös-kulturellen Filter, kostenlos Getränke und Obst als ergänzender Anreiz sowie schließlich die freie Zeit des Wochenendes; alles freiwillig und überwiegend selbstgesteuert.

\section{Schablone für andere Projekte}

Diese komplexen Angebote müssen professionell organisiert und koordiniert werden, da andernfalls die Last des Erfolges ein derartiges Sozialprojekt scheitern lässt. Hier wäre Open Sunday nicht das erste und wohl auch nicht das letzte, dem es so erginge. Folgerichtig werden dezidiert "Gelingensbedingungen“ formuliert, damit die Leser*innen eine anschauliche Schablone zur Verfügung haben, selbst die eigenen Projekte daran zu spiegeln oder aber ein ähnliches Vorhaben mit den bewährten Erfahrungen der Autoren*innen erfolgreich initiieren zu können. Diese Gelingensbedingungen reichen vom institutionellen Zusammenspiel - Kooperation von Grundschulen mit Sportvereinen samt Stadtsportbünden, den kommunalen Integrationszentren sowie der Alma Mater der Autor*innen selbst - bis hin zu den personellen Fragen: Welche Aufgaben müssen die Sozialarbeiter*innen übernehmen, welche Rolle tragen die jugendlichen Sporthelfer*innen als Mentor ${ }^{\star}$ innen, wo können Sportstudierende und Übungsleiter*innen ihre Expertise beisteuern? Diese operativen Fragen sind es, die das Buch so reizvoll machen, da ihre Antworten über das Wohl und Wehe eines sportpädagogischen Projekts Sozialer Arbeit auf der konkreten Handlungsebene entscheiden.

Das Buch zeigt mit Hilfe von vielen Tabellen und Grafiken übersichtlich auf, wie sich das Projekt des Open Sunday zunehmend professionalisiert und erweitert hat. Die Expansion geht mit den typischen Begleiterscheinun- 


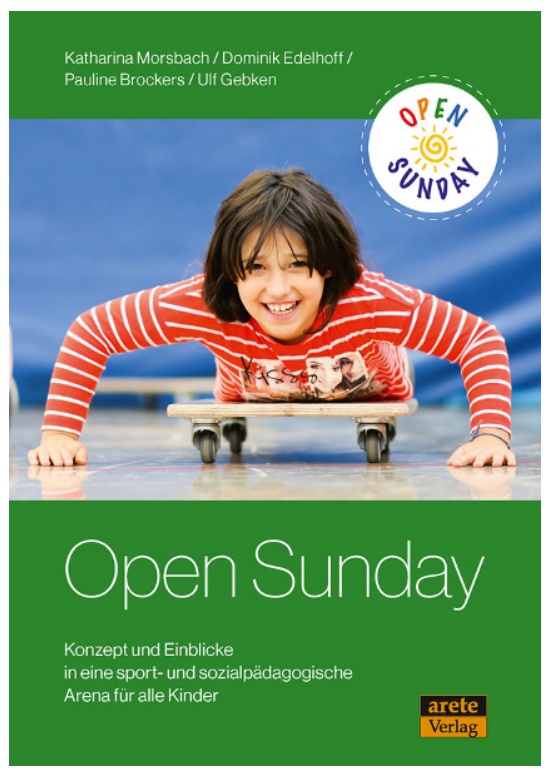

Abb. 1 \& In "Open Sunday" stellen die Autor*innen ein sportpädagogisches Konzept für eine offene Kinder- und Jugendarbeit in herausfordernden Stadtteilen vor. (Abbildung: Arete Verlag)

gen eines mehrdimensionalen Scalingup-Konzepts einher, bei dem die Tiefe der Entwicklung, die bereits erwähnte Nachhaltigkeit, die Verbreitung und der Wechsel der Reformträger ausschlaggebend sind. Erfreulicherweise ehrlich sind die Autoren*innen dann auch, wenn sie in sehr selbstkritischer Weise von den noch vorhandenen Einschränkungen, Schwierigkeiten, aber auch noch zu leistenden Aufgaben berichten. Gleichzeitig spannt das Buch aber gerade deshalb zukünftige Perspektiven nicht nur für eine bloße Weiter-, sondern für die Besserentwicklung auf.

\section{Valider Nachweis fehlt noch}

Sucht man nämlich über die subjektiv wahrgenommenen Gelingensbedingungen hinaus nach weiteren faktischen Erfolgsfaktoren des Open Sunday, findet man neben der hohen Erreichbarkeitsziffer nur bedingt belastbare Daten zu den empirisch-analytischen Effekten. Dies ist dem Forschungsansatz des Open Sunday geschuldet, der sich nach Kahlert (2015) als "pragmatische Forschung“ zusammenfassen lässt. Zentraler Leitgedanke dieses Ansatzes ist, dass „Erkenntnisse nicht zwingend aus experimentellen
Studien generiert werden müssen, sondern ebenfalls aus den unmittelbaren sozialen Handlungsvollzügen" (S. 83). Erkenntnistheoretisch ist dies die wunde Stelle von Open Sunday, da der „blinde Fleck" zwar durch Selbstreflexion und reziproke Rückkopplungsschleifen der Beteiligten verkleinert, aber nicht beseitigt werden kann. Als konkreter Vorschlag an die Autoren ${ }^{*}$ innen sei eine längsschnittlich durchzuführende Effektestudie genannt, welche die im Buch vielfältig reklamierten Zielsetzungen in einem randomisierten und kontrollierten Design durch eine externe Evaluation untersuchen lässt. Hier sollten die Autoren*innen auch mutiger sein, denn qualitativ ist das Projekt ein großer Fundus an wertvollen Ideen und Ansätzen. Die Wirkungsannahmen sind logisch hypothetisiert und das Rahmenkonstrukt ist plausibel theoretisiert. Allein, es fehlt der objektiv valide Nachweis, damit der selbst angenommene Impact belegt werden kann. Aber das ahnen die Autoren*innen auch aufgrund der in Kap. 5 formulierten epistemologischen Selbstkritik offenkundig.

Das Buch schließt mit einem Blick in die Zukunft, die jetzt schon prosperiert. Mit dem „Mini Sunday“ und der „Open Arena" haben sich bereits Tochterideen durchgesetzt, bei denen im ersten Fall Zwei- bis Sechsjährige die Zielgruppe sind und im zweiten Falle Jugendliche. Open Sunday hat sich also mit Blick auf die Altersgruppen stark verbreitert. Deshalb ist es konsequent, dass die Projektverantwortlichen ein Starterpaket entwickelt haben mit Schulungen für Interessierte, die das professionelle Niveau absichern helfen. Als Modell (sehr) guter Praxis für sportbezogene Kinder- und Jugendarbeit sei deshalb der „offene Sonntag" als Lektüre empfohlen.

\section{Korrespondenzadresse}

\section{Prof. Dr. Rolf Schwarz}

Institut für Bewegungserziehung und Sport, Pädagogische Hochschule Karlsruhe

Karlsruhe, Deutschland

rolf.schwarz@ph-karlsruhe.de

Rolf Schwarz ist an der Pädagogischen Hochschule Karlsruhe Leiter des Studiengangs Sport-GesundheitFreizeitbildung und Bereichsleiter für Bewegungs- bildung im Studiengang Pädagogik der Kindheit. Zu seinen Forschungsschwerpunkten zählt die entwicklungspädagogische Optimierung von Bewegungs-, Spiel- und Sporträumen.

Funding. Open Access funding enabled and organized by Projekt DEAL.

Open Access. Dieser Artikel wird unter der Creative Commons Namensnennung 4.0 International Lizenz veröffentlicht, welche die Nutzung, Vervielfältigung, Bearbeitung, Verbreitung und Wiedergabe in jeglichem Medium und Format erlaubt, sofern Sie den/die ursprünglichen Autor(en) und die Quelle ordnungsgemäß nennen, einen Link zur Creative Commons Lizenz beifügen und angeben, ob Änderungen vorgenommen wurden.

Die in diesem Artikel enthaltenen Bilder und sonstiges Drittmaterial unterliegen ebenfalls der genannten Creative Commons Lizenz, sofern sich aus der Abbildungslegende nichts anderes ergibt. Sofern das betreffende Material nicht unter der genannten Creative Commons Lizenz steht und die betreffende Handlung nicht nach gesetzlichen Vorschriften erlaubt ist, ist für die oben aufgeführten Weiterverwendungen des Materials die Einwilligung des jeweiligen Rechteinhabers einzuholen.

Weitere Details zur Lizenz entnehmen Sie bitte der Lizenzinformation auf http://creativecommons.org/ licenses/by/4.0/deed.de.

\section{Literatur}

Kahlert, J. (2015). Pragmatik. In J. Kahlert, M. FöllingAlbers, M. Götz, A. Hartinger, S. Miller \& S. Wittkowske (Hrsg.), Handbuch Didaktik des Sachunterrichts (2. Aufl. S.51-55). Bad Heilbrunn: Klinkhardt. 